\title{
Análisis Longitudinal del Rendimiento Escolar según el Compromiso Escolar y Factores Contextuales: El Caso Chileno
}

\author{
Longitudinal Analysis of School Performance According to School Engagement and \\ Contextual Factors: The Chilean Case
}

\author{
Horacio Miranda ${ }^{1}$, Edgardo Miranda-Zapata ${ }^{2}$ Laura Lara $^{3}$ y Mahia Saracostti ${ }^{4}$
}

\begin{abstract}
Resumen
Con la finalidad de cuantificar el efecto de factores contextuales sobre el compromiso escolar y las variables de rendimiento escolar: promedio de notas y asistencia a clases en escolares chilenos evaluados en dos momentos en el tiempo, se elaboró un modelo de ecuaciones estructurales (SEM) longitudinal que relaciona las subdimensiones de factores contextuales (familia, profesorado y pares) y compromiso escolar (afectivo, conductual, cognitivo) con el rendimiento escolar. Se seleccionaron las subdimensiones de factores contextuales y compromiso escolar más explicativas del promedio de notas y asistencia a clases, quedando fuera el compromiso escolar cognitivo. El modelo SEM longitudinal presentó buena bondad de ajuste (RMSEA=.034; $\mathrm{CFI}=.929$; TLI=.926). Los análisis comparativos detectaron diferencias significativas entre combinaciones de subdimensiones de factores contextuales y compromiso escolar tanto transversalmente como por trayectorias de promedio de notas y asistencia a clases a través del tiempo.
\end{abstract}

Palabras clave: análisis longitudinal, trayectoria educativa, compromiso escolar, rendimiento escolar

\begin{abstract}
In order to quantify the effect of contextual factors on school engagement and school performance variables: grade point average (GPA) and class attendance in Chilean schoolchildren evaluated at two moments in time, a longitudinal structural equation model (SEM) was developed that relates the subdimensions of contextual factors (family, teachers and peers) and school engagement (affective, behavioral, cognitive) with school performance. The most explanatory sub-dimensions of contextual factors and school engagement of GPA and class attendance were selected, so cognitive school engagement was not included. The longitudinal SEM model showed good goodness-of-fit (RMSEA=.034; CFI=.929; TLI=.926). The comparative analysis showed significant differences of GPA and class attendance by combinations of contextual factors and school engagement sub-dimensions both transverse and over time.
\end{abstract}

Keywords: longitudinal analysis, educational trajectory, school engagement, school performance

\footnotetext{
${ }^{1}$ Magíster en Bioestadística. Académico e investigador. Departamento de Producción Agropecuaria, Universidad de La Frontera. Universidad de la Frontera, Avenida Francisco Salazar, 01145. Temuco, Chile. Tel.: + 56 452734167. Correo: horacio.miranda@ufrontera.cl ${ }^{2}$ Doctor en Metodología de las Ciencias del Comportamiento y de la Salud. Académico e Investigador. Núcleo Científico Tecnológico en Ciencias Sociales y Humanidades, Universidad de La Frontera. Universidad de la Frontera, Avenida Francisco Salazar, 01145. Temuco, Chile. Tel.: +56 452596732. Correo: edgardo.miranda@ufrontera.cl

${ }^{3}$ Laura Lara. Doctora en Psicología. Profesora Titular. Universidad Autónoma de Chile, 5 poniente 1670, Talca, Chile. Tel.: +56712735770. Correo: 1larav@uautonoma.cl

${ }^{4}$ Doctora en Social Welfare. Profesora Titular de la Escuela de Trabajo Social de la Facultad de Ciencias Sociales. Directora de la Cátedra UNESCO Niñez, Juventud, Educación y Sociedad. Universidad de Valparaíso. Universidad de Valparaíso, Blanco 951, Valparaíso, Chile. Tel.: +56998293161. Correo: mahia.saracostti@uv.cl
} 


\section{Introducción}

El logro de aprendizajes evaluado como rendimiento escolar y la presentación de trayectorias educativas exitosas son características asociadas en forma significativa con el Compromiso Escolar (CE) siendo este un componente clave para prevenir la deserción escolar, dado que la decisión de abandonar la escuela es un consecuente final de un proceso gradual de pérdida de interés y vinculación con los estudios. $\mathrm{El} \mathrm{CE}$ es una variable multidimensional y se compone de tres subdimensiones: el compromiso cognitivo, afectivo y conductual.

La Constitución Chilena determina la obligatoriedad de la educación básica y media, sin embargo, en base a las cifras aportadas por el Centro de Estudios MINEDUC (2014), la tasa de deserción escolar se aproxima al 3,1\% principalmente en $1^{\circ}$ de educación media. Según datos de la CEPAL (2015), la tasa de deserción en la enseñanza secundaria en Chile sería aún mayor, equivalente al $10 \%$, si se considera el rezago escolar como factor de riesgo de deserción escolar, donde existen cerca de 155.000 estudiantes menores de 17 años, con dos o más años de regazo y casi 370.000 con un año de rezago (ACHM et al., 2013). Además, estas cifras se concentran principalmente en estudiantes que pertenecen al quintil de más bajos recursos económicos que asisten a establecimientos educacionales municipales (ACHNU, 2006; Espinoza-Díaz et al., 2014).

La revisión evidencia los componentes que conforman el $\mathrm{CE}$, en general apunta a que familiares, compañeros/as y profesores/as son los tres contextos relacionales principales asociados con la participación escolar. El estudio realizado por Fernández-Zabala, Goñi y Zulaika (2015), muestra que además existen otros componentes relevantes como el apoyo percibido de los pares, así como en compromiso conductual y emocional.

El estudio longitudinal sobre las trayectorias y patrones del compromiso escolar, realizado por Wylie y Hodgen (2012), muestra como los patrones de compromiso arrojan información útil acerca de los factores que contribuyen al desempeño escolar en función de diferentes niveles de participación e implicación de los y las estudiantes en la escuela. En la misma línea, Gorostiaga, Balluerka, Ulaciay y Aliri (2017) establecen la relevancia dl rol de la iniciativa y su función sobre el rendimiento académico de los estudiantes.

La evidencia propone revisar la trayectoria educativa de los y las estudiantes y las dimensiones asociadas a la misma. En este sentido, Melo, Failache y Machado (2015) ponen de relieve la importancia del contexto familiar en dichas trayectorias, reportando que dos tercios de los adolescentes uruguayos que participaron en su estudio dejaron de asistir al sistema educativo formal cuando tenían 14 años o menos y el $40 \%$ nunca se matriculó en Educación Media. La decisión de deserción escolar de la mayor parte de estos adolescentes fue apoyada por la familia.

Respecto a los estudios desarrollados en Chile, Espinoza-Díaz, Castillo-Guajardo, González, Loyola-Campos y Cruz-Grau (2014) identifican importantes diferencias en las evoluciones educativas de estudiantes que desertan del sistema educativo y los y las estudiantes que no entregando antecedentes sobre acciones que se podrían realizar en la escuela para prevenir la deserción escolar temprana. La mayoría de los y las estudiantes que desertan del sistema escolar evidencian características similares como bajo rendimiento académico, repeticiones de curso, mal comportamiento, inasistencia a clases y frecuentes cambios de escuela, las cuales podría confluir como limitantes en las destrezas socio-adaptativas de los estudiantes (Vera-Bachmann, Gálvez-Nieto, Trizano Hermosilla, \& Álvarez-Espinoza, 2020).

Este estudio tiene como objetivo analizar los cambios en el tiempo (medido en dos momentos) de la influencia del compromiso escolar y los factores contextuales sobre el promedio de notas y la asistencia a clases Debido a que la evidencia reporta que el $\mathrm{CE}$ se relaciona positivamente con el éxito de las trayectorias educativas tales como el rendimiento escolar (promedio de notas) y el porcentaje de asistencia a clases, este estudio se propone evaluar la calidad de medición de un modelo de ecuaciones estructurales (SEM) de tipo longitudinal conformado por las dimensiones del CE tales (afectiva, cognitiva y conductual) además de factores Contextuales (familia, profesorado y pares) incluyendo como variables 
dependientes el desempeño de aprendizaje escolar y la asistencia a clases (Figura 1). Por lo tanto,

Las hipótesis planteadas son tres. La primera plantea que el modelo SEM longitudinal del FC y CE presentará buenos indicadores de precisión y bondad de ajuste a los datos observados en la muestra. Con la finalidad de analizar la significancia de la diferencia de los rendimientos escolares medidos como la resta del PN así como del porcentaje anual de AC entre los dos momentos en el tiempo. La segunda hipótesis corresponde a la comparación transversal de la resta de los rendimientos observados entre dos momentos en el tiempo, que busca evidenciar significancia estadística de la diferencia de rendimiento en $\mathrm{PN}$ y $\mathrm{AC}$ entre las condiciones determinadas por las variables de CE y contextuales tanto en el grupo de estudiantes que mejoraron o mantuvieron su rendimiento escolar en el tiempo, así como en el grupo de estudiantes que disminuyeron su rendimiento escolar a través del tiempo. Finalmente, la tercera hipótesis plantea evidenciar diferencias significativas en las trayectorias del PN y AC entre las condiciones determinadas por las subdimensiones de FC y CE tanto en el grupo de estudiantes que mejoraron o mantuvieron su rendimiento escolar, así como en el grupo de estudiantes que disminuyeron su rendimiento escolar a través del tiempo.

El cumplimiento de las hipótesis planteadas en este estudio permitirá profundizar en las magnitudes de las diferencias transversales y las trayectorias longitudinales del rendimiento escolar, así como en las características de las subdimensiones de FC y CE que correspondieron a los y las estudiantes que presentaron un aumento o mantención de la diferencia o las trayectorias de $\mathrm{PN}$ y $\mathrm{AC}, \mathrm{y}$ en los y las estudiantes que presentaron disminución de sus rendimientos a través del tiempo.

\section{Método}

El estudio es no experimental con diseño longitudinal correlacional-causal, basado en unidades de muestreo que presentan información auto-correlacionada en el tiempo. En concordancia con incluir un análisis de constructos latentes medidos a través del tiempo, se aplica una modelización de ecuaciones estructurales de tipo longitudinal que permite evaluar apropiadamente la bondad de ajuste y la adecuación del modelo a los datos empíricos recogidos en la muestra.

\section{Participantes}

La muestra incluye 271 estudiantes de $7^{\circ}$ Básico a $1^{\circ}$ Medio de colegios municipales de alta vulnerabilidad (JUNAEB, 2015), pertenecientes a dos regiones de Chile (Libertador Bernardo O'Higgins y La Araucanía). El $45.5 \%$ fueron chicos y $54.5 \%$ chicas, con edades comprendidas entre los 12 y los 16 años, siendo el promedio los 14 años de edad. La estructura de la muestra corresponde a un diseño no probabilístico por conveniencia, aplicado en dos años consecutivos. Los cuestionarios analizados correspondieron a los y las participantes que contaban con los respectivos asentimientos y consentimientos informados, con respuestas completas, no constantes en los ítems de las seis dimensiones y asociados con los correspondientes datos de promedio de notas y porcentaje de asistencia a clases en ambos momentos en el tiempo.

\section{Instrumentos}

Cuestionario de Compromiso Escolar. Desarrollado por Lara et al. (2018), es un instrumento que consta de 29 ítems en escala tipo Likert de cinco puntos (desde 1, Muy en desacuerdo, hasta 5, Muy de acuerdo), que avalúa el Compromiso Escolar desde su concepción tridimensional: Compromiso Afectivo (10 ítems), Compromiso Conductual ( 7 ítems) y Compromiso Cognitivo (12 ítems). El instrumento presenta buenos índices de fiabilidad como consistencia interna para cada subescala (Compromiso Afectivo: $\omega=.877$ y $\alpha=.849$, Compromiso Conductual: $\omega=.827$ y $\alpha=.692$, Compromiso Cognitivo: $\omega=.904$ y $\alpha=.883)$ y evidencia a favor de la validez de constructo $\left(X^{2}=2145.09 ; g l=374\right.$; RMSEA=.054; CFI=.915; TLI=.907).

Cuestionario de Factores Contextuales. Registrado por la Universidad de La Frontera y la Universidad Autónoma de Chile (2016), es un instrumento que consta de 18 ítems en escala tipo Likert de 5 puntos (desde 1, Muy en desacuerdo, hasta 5, Muy de acuerdo), que evalúa variables de contexto, consistente en la interacción con personas del entorno de los y las estudiantes: 


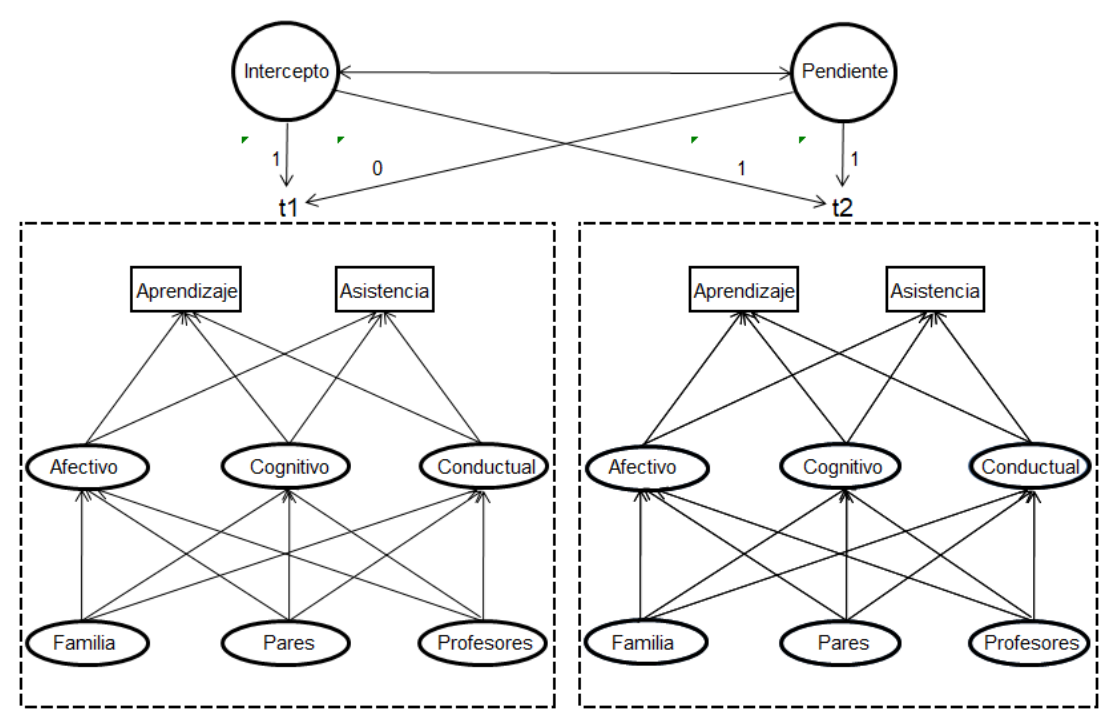

Figura 1. Esquema simplificado del modelo SEM longitudinal

Familia (3 ítems), Profesorado (8 ítems) y Pares (7 ítems). El instrumento presenta buenos índices de fiabilidad como consistencia interna para cada subescala (Familia: $\omega=.801$ y $\alpha=.735$, Profesorado: $\omega=.907$ y $\alpha=.884$, Pares: $\omega=.864$ y $\alpha=.889)$ y evidencia a favor de la validez de constructo $\left(X^{2}=728.72 ; g l=132 ;\right.$ RMSEA $=.053$; CFI=.969; TLI=.964).

\section{Procedimiento}

El acceso a los colegios y la aplicación de los instrumentos fue autorizado por los directivos de los establecimientos y los apoderados mediante una firma suscrita en los correspondientes consentimientos informados que fueron previamente examinados y aprobados por el Comité de Ética Científica de la Universidad de La Frontera, Temuco, Chile. En el momento de responder los cuestionarios los y las estudiantes firmaron un documento de asentimiento que confirmaba su participación bajo ningún tipo de coerción y habiendo sido informados de los propósitos y el resguardo de la confidencialidad y el secreto estadístico de las respuestas entregadas a los investigadores. En forma complementaria a la información recogida, las personas encargadas de los establecimientos también entregaron los datos del rendimiento escolar medido como el promedio de nota y el porcentaje de asistencia a clases de los y las estudiantes.

\section{Plan de análisis}

La información analizada considera aquellos registros que presentan información completa en las seis dimensiones (las que conforma en compromiso escolar y las tres que conforman los factores contextuales) y las variables de rendimiento escolar estudiadas en dos momentos en el tiempo, esto totaliza 271 casos lo que determina una matriz de información conformada por 3252 datos.

El modelo SEM analizado en cada momento en el tiempo corresponde al reportado por Miranda-Zapata, Lara, Navarro, Saracostti y De Toro (2018), que presentó propiedades psicométricas apropiadas (RMSEA=.046; CFI=.919; TLI=.912). De este modo, tomamos como valores de referencia para indicar un ajuste aceptable un RMSEA menor que .06 (Marsh, Hau, \& Grayson, 2005), y valores CFI y TLI mayores que .90 (Kline, 2010). El modelo de ecuaciones estructurales longitudinal fue analizado mediante el programa Mplus 7.3 utilizando la matriz de correlaciones policóricas y el método de estimación de parámetros de cuadrados mínimos no ponderados robustos (ULSMV). El diagrama simplificado del modelo SEM longitudinal orientado a mostrar las variables incluidas en los tiempos $\mathrm{t} 1$ y t 2 se presenta en la Figura 1.

Los análisis estadísticos comparativos de tipo transversal emplean como variable dependiente la diferencia entre el rendimiento escolar del momento final menos el rendimiento observado en el momento inicial en PN y AC, esto permite separar los rendimientos de los y las estudiantes en dos grupos: estudiantes que presentan una diferencia con valor positivo o cero que representa a los estudiantes que mejoran o mantienen su 
rendimiento escolar entre los dos momentos en el tiempo y estudiantes que presentan una diferencia con signo negativo que corresponde a los estudiantes que disminuyen su rendimiento escolar en el tiempo. Este procedimiento permite obtener dos variables calculadas, una para el diferencial del PN y otra para $\mathrm{AC}$, que al ser dicotomizadas de acuerdo a sus signos se usaron como variables de control para realizar análisis separados por grupos, tanto para el grupo de estudiantes que aumentan o mantienen sus rendimientos escolares en el tiempo (variables de control con categoría correspondiente a signo positivo: +), así como para el grupo de estudiantes que disminuyen sus rendimientos en el tiempo (variables de control con categoría de signo negativo: -). Lo anterior determina la conformación de cuatro grupos de datos compuestos por las variables de rendimiento escolar $\mathrm{PN}$ y $\mathrm{AC}$ y las categorías de rendimiento (+) y (-).

Para la conformación de segmentos comparativos predictivos de las diferencias del PN y AC entre los momentos en el tiempo, se emplea un análisis de árbol de decisión mediante la técnica de árboles de clasificación y regresión (CART) para identificar las combinaciones de subdimensiones de FC y CE con mayor grado de explicación de las diferencias observadas en los rendimientos escolares a través del tiempo.

El análisis CART es aplicado separadamente en los cuatro grupos de datos correspondientes a las variables NP y AC y los niveles de desempeños (+) y (-). De esta forma, se obtienen segmentos conformados por categorías determinadas mediante puntos de cortes binarios establecidos sobre combinaciones de subdimensiones de FC y CE. Estos segmentos corresponden a las denominadas "hojas" del árbol de regresión, que se obtienen mediante particiones secuenciales del árbol de decisión hasta conformar tres segmentos correspondientes a niveles alto, medio y bajo en NP y AC separadamente. Estas categorías CART son agregadas a los datos para ser empleadas como factores predictores en análisis comparativos del rendimiento escolar de PN y AC dentro de los grupos de desempeños mayor (+) y menor (-), determinando un total de cuatro secciones de análisis de datos tanto para los análisis comparativos transversales como longitudinales.
Para realizar los análisis comparativos de tipo transversal, los segmentos CART son incluidos como variable independiente en un análisis de varianza (ANOVA) de un factor, con la finalidad de comparar estadísticamente la diferencia de los rendimientos de NP y AC entre los segmentos resultantes de la combinación de las subdimensiones de FC y CE. Para los casos que presentan diferencias significativas en el ANOVA, se aplican comparaciones múltiples de tipo paramétrico (prueba HSD de Tukey), con la finalidad de identificar y caracterizar los segmentos que presentan diferencias estadísticamente significativas tanto en el grupo de estudiantes que mejoran o mantienen su rendimiento escolar, así como en el grupo de estudiantes que disminuyen sus rendimientos escolares con respecto al momento inicial.

Para los análisis longitudinales de comparación de trayectorias de las variables de PN y AC, se emplea un análisis de varianza multivariante de medidas repetidas con la finalidad de estimar diferencias significativas entre los segmentos CART. Los casos que presentan diferencias significativas continúan con una prueba de comparación múltiple de contrastes ortogonales, para identificar perfiles de segmentos que unen trayectorias similares de rendimiento escolar, tanto en el grupo de estudiantes que mejoran o mantienen su rendimiento escolar, así como para el grupo de estudiantes que disminuyen su rendimiento en el tiempo.

\section{Resultados}

\section{Bondad de ajuste y precisión del modelo SEM longitudinal}

La estructura del modelo de ecuaciones estructurales longitudinal incluye los factores de contexto correspondientes a las subdimensiones de FC: familiar, pares y profesorado, que operan como antecedentes sobre las subdimensiones del compromiso escolar: afectivo, conductual y cognitivo, que a su vez actúan como efecto mediador completo sobre las variables objetivas o nodos finales del modelo correspondientes al rendimiento expresado como la nota y el porcentaje de asistencia a clases de los estudiantes evaluados en dos momentos en el tiempo. 
Tabla 1. Diferencia de promedio de notas $\left(\mathrm{t}_{2}-\mathrm{t}_{1}\right)$ en estudiantes rendimientos escolares positivos y negativos en el tiempo

\begin{tabular}{lcccccc}
\hline Grupo & \multicolumn{2}{c}{ Mejoran o mantienen nota (+) } & \multicolumn{3}{c}{ Disminuyen nota (-) } \\
\hline Diferencia ( $\left.\mathrm{t}_{2}-\mathrm{t}_{1}\right)$ & Mayor & Medio & Menor & Mayor & Medio & Menor \\
\hline Promedio nota (sig.) & $.27(\mathrm{a})$ & $.20(\mathrm{a})$ & $.07(\mathrm{~b})$ & $-.30(\mathrm{c})$ & $-.19(\mathrm{~d})$ & $-.12(\mathrm{~d})$ \\
Segmento CART & Familia & Familia & Profesor & Afectivo & Familia & Familia \\
(participación) & (menor) & (mayor) & (menor) & (menor) & (menor) & (mayor) \\
\hline
\end{tabular}

La comparación entre segmentos CART (Mayor, Medio, Menor) dentro de grupos (+) y (-),presentó diferencias significativas ( $p \leq .05)$. Letras distintas entre paréntesis representan diferencias significativas en la prueba de Tukey $(p \leq .05)$.

Para medir las propiedades psicométricas del modelo SEM, se estimaron los parámetros considerando la naturaleza ordinal de las variables indicadoras. La totalidad de las cargas del modelo presentaron estimaciones estadísticamente significativas $(p<.001)$. El resumen de las significancias estadísticas de los principales parámetros estimados de modelo se presenta en Anexos.

La evaluación de la calidad del ajuste del modelo presentó una razón entre el valor ChiCuadrado sobre los grados de libertad menor que 2, lo que corresponde a una evaluación indirecta favorable en cuanto a la bondad de ajuste global del modelo $\left(X^{2}=5703 ; g l=4371\right)$.

En concordancia con lo anterior se obtuvo un RMSEA=.034, evidenciando que el modelo alcanza un buen nivel de precisión entre la matriz de información empírica y la matriz de representación teórica del modelo evaluado.

Con respecto a los índices de ajuste incremental del modelo, estos correspondieron a $\mathrm{CFI}=.929$ y TLI=.926, lo que evidencia un buen nivel de bondad de ajuste del modelo propuesto con base en la información empírica proveniente de la muestra estudiada. De este modo, los datos corroboran la aceptación de la primera hipótesis, ya que fue posible evidenciar una alta calidad de ajuste y precisión del modelo longitudinal, obteniéndose estimaciones de parámetros altamente significativas con estructural longitudinal de los factores del compromiso escolar y contextual sobre la diferencia de PN y AC en el tiempo.

\section{Análisis transversal y longitudinal del rendimiento escolar en el tiempo}

Las variables de respuesta empleadas en el análisis transversal correspondieron a la diferencia del rendimiento escolar entre el momento final menos el inicial. En el caso del análisis longitudinal las variables de respuesta correspondieron a los valores de promedio de nota y porcentaje de asistencia observados en cada momento. Todos los análisis se realizaron por grupos separados según los y las estudiantes que mejoraron o mantuvieron su rendimiento escolar, separados del grupo que presentó disminución en las variables de respuesta.

\section{Análisis transversal de la diferencia del promedio de notas en el tiempo}

Para analizar el rendimiento escolar del promedio de notas, se comparan los segmentos CART correspondientes a las combinaciones de las subdimensiones de $\mathrm{CE}$ y FC, estos presentaron diferencias estadísticamente significativas tanto en el análisis de varianza como en las pruebas de comparación múltiple de promedios de Tukey ( $p$ $<.05)$. Los segmentos estadísticamente diferentes se representan mediante letras distintas al interior de la tabla de resultados. Los análisis se realizaron tanto para el grupo que mejoró o mantuvo su promedio de nota así como en el grupo que disminuyó (Tabla 1).

$\mathrm{Al}$ focalizar el análisis en los niveles de menor y mayor diferencia de promedio de notas, se observa que el menor nivel de notas se relaciona con un menor compromiso escolar afectivo, lo que podría prever una posible situación de indiferencia de este tipo de estudiantes con respecto a su entorno educativo, en cambio el mayor rendimiento de notas se presentó asociado con una menor participación del factor contextual familiar, los resultados de este último caso corresponderían a una situación de mayor autonomía por parte de este tipo de estudiantes. Cabe hacer notar que el factor contextual familiar se presenta como un factor de soporte para el segmento de nivel medio de mejoramiento de la nota, y además este factor actuaría a través de un efecto mediado por el componente del compromiso escolar. 
Tabla 2. Diferencia de asistencia $\left(\mathrm{t}_{2}-\mathrm{t}_{1}\right) \%$ en estudiantes con logros positivos y negativos en el tiempo

\begin{tabular}{lcccccc}
\hline Grupo & \multicolumn{2}{c}{ Mejoran o mantienen asistencia (+) } & \multicolumn{3}{c}{ Disminuyen asistencia (-) } \\
\hline Diferencia $t_{2}-\mathrm{t}_{1}$ & Mayor & Media & Menor & Mayor & Media & Menor \\
\hline Asistencia (sig.) & $6.20(\mathrm{a})$ & $4.60(\mathrm{a})$ & $1.85(\mathrm{~b})$ & $-4.23(\mathrm{c})$ & $-3.78(\mathrm{c})$ & $-2.21(\mathrm{~d})$ \\
Segmento CART & Afectivo & Pares & Pares & Profesor & Conductual & Profesor \\
(participación) & (mayor) & (mayor) & (menor) & (mayor) & (mayor) & (menor) \\
\hline
\end{tabular}

La comparación entre segmentos CART (Mayor, Medio, Menor) dentro de grupos (+) y (-),

presentó diferencias significativas ( $p \leq .05)$. Letras distintas representan diferencias significativas en la prueba de Tukey ( $p \leq .05)$.

\section{Análisis transversal de la diferencia de asistencia a clase en el tiempo}

En este análisis la variable de respuesta correspondió a la diferencia del porcentaje de asistencia del segundo año del estudio menos lo observado en el año anterior. Los grupos comparados corresponden a los segmentos CART determinados por las combinaciones de las subdimensiones de $\mathrm{CE}$ y $\mathrm{FC}$, estos presentaron diferencias estadísticamente significativas tanto en el análisis de varianza como en las pruebas de comparación múltiple de promedios de Tukey $(p<.05)$. Los segmentos estadísticamente diferentes se representan mediante letras distintas al interior de la tabla de resultados. Los análisis se realizaron en forma separada para los estudiantes que mejoraron o mantuvieron su asistencia, así como para aquellos y aquellas que disminuyeron su asistencia en el tiempo, los resultados se presentan en la Tabla 2.

Al focalizar el análisis en los extremos de mayor y menor asistencia a clases se observa que el nivel de mayor rendimiento se encuentra asociado al compromiso escolar afectivo y la mediación de los pares, en cambio el menor nivel de asistencia se encuentra relacionado con un mayor énfasis del compromiso escolar conductual y una mayor participación del factor contextual del profesorado, se debe considerar que este último efecto se encuentra mediado por el componente del compromiso escolar. En relación con el objetivo 2, se puede concluir que éste se cumplió a favor de la hipótesis de trabajo planteada ya que fue posible evidenciar diferencias estadísticamente significativas en el análisis transversal de los factores del compromiso escolar y contextual sobre la diferencia de PN y AC en el tiempo.

\section{Análisis longitudinal de las trayectorias de rendimiento escolar}

Para realizar el análisis comparativo longitudinal de las trayectorias del promedio de notas y el porcentaje de asistencia a clases entre los segmentos CART, se procedió a aplicar un análisis de varianza multivariante longitudinal de medidas repetidas sobre los rendimientos observados. En los casos que se presentaban diferencias significativas, se procedió a realizar una prueba de comparación múltiple de contrastes ortogonales para identificar los segmentos que presentaban trayectorias estadísticamente diferentes. Este análisis se realizó en forma separada por los grupos de estudiantes que aumentaron o disminuyeron su rendimiento escolar a través del tiempo.

\section{Análisis longitudinal de las trayectorias del promedio de las notas a través de tiempo}

Para realizar el análisis de las trayectorias del promedio de notas, en el grupo de estudiantes que mejoraron o mantuvieron su nota, se aplicó el test multivariante de Wald el cual permitió determinar que existían diferencias estadísticamente significativas entre las tendencias de las trayectorias a través del tiempo ( $p<.001)$ (Figura 2).

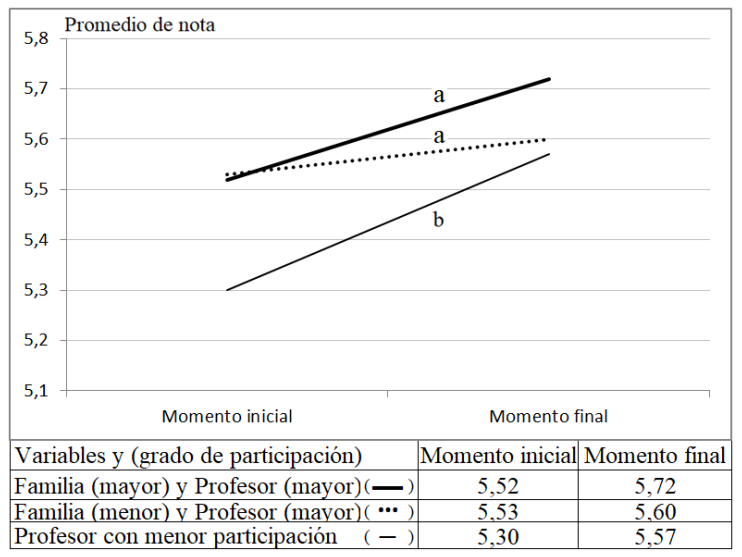

Figura 2. Gráfico de trayectorias de promedio de notas del grupo que aumenta o mantiene su promedio en el tiempo 
La aplicación del test de contrastes ortogonales evidenció que las trayectorias de los segmentos CART conformaron dos subpoblaciones (a $\quad$ y $\quad$ b) significativamente diferentes entre sí $(p \leq .05)$. Presentando la menor respuesta el segmento con menor participación del profesorado, en cambio las trayectorias de mejor desempeño corresponden a las relacionadas con el factor contextual de la familia y el profesorado, esta significancia fue estimada a través de un efecto mediado por el componente del compromiso escolar. De este modo, los datos corroboran la segunda hipótesis, ya que fue posible evidenciar diferencias estadísticamente significativas en el análisis transversal de los factores del compromiso escolar y contextual sobre la diferencia de PN y AC en el tiempo.

El análisis de las trayectorias del promedio de notas en el grupo de estudiantes que disminuyen su respuesta a través del tiempo, el test multivariante de Wald determinó que existían diferencias significativas entre las tendencias de las trayectorias en el tiempo ( $p<.001)$ (Figura 3).

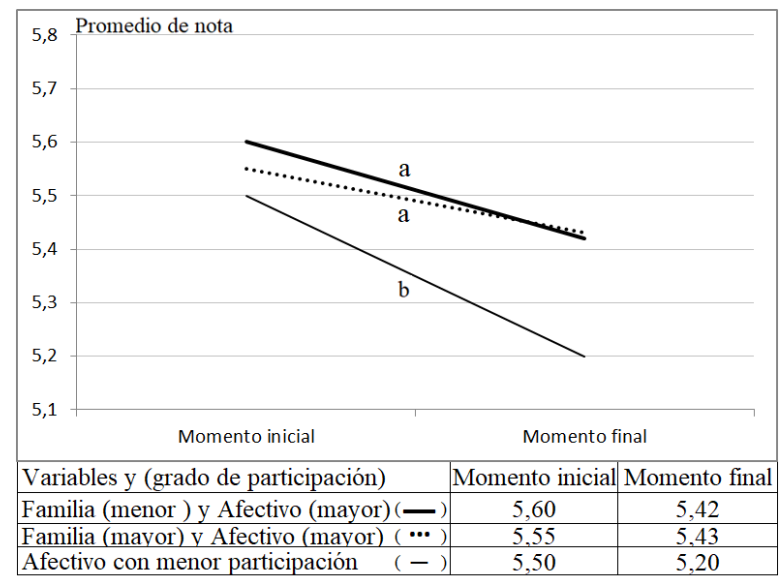

Figura 3. Gráfico de trayectorias de promedio de notas del grupo que disminuye su promedio en el tiempo

La aplicación del test de contrastes ortogonales determinó que las trayectorias de los segmentos CART conformaban dos subpoblaciones ( $\left.\begin{array}{lll}a & y & b\end{array}\right)$ significativamente diferentes entre sí ( $p<.05)$. Presentando la menor respuesta el segmento de estudiantes con menor compromiso afectivo. Las trayectorias que presentaron una tendencia hacia el equilibrio correspondieron a aquellas asociadas con el compromiso afectivo y una mayor participación del factor contextual relacionado con el profesorado. Cabe mencionar que la significancia de este factor contextual se encuentra mediado por el componente del compromiso escolar.

\section{Análisis longitudinal de las trayectorias de la asistencia a clases a través de tiempo}

El análisis de las trayectorias del porcentaje de asistencia según los segmentos de las subdimensiones de FC y $\mathrm{CE}$, en el grupo de estudiantes que aumentaron o mantuvieron su asistencia a clases, el test multivariante de Wald mostró que existían diferencias estadísticamente significativas entre las tendencias de las trayectorias a través del tiempo ( $p<.001)$ (Figura 4).

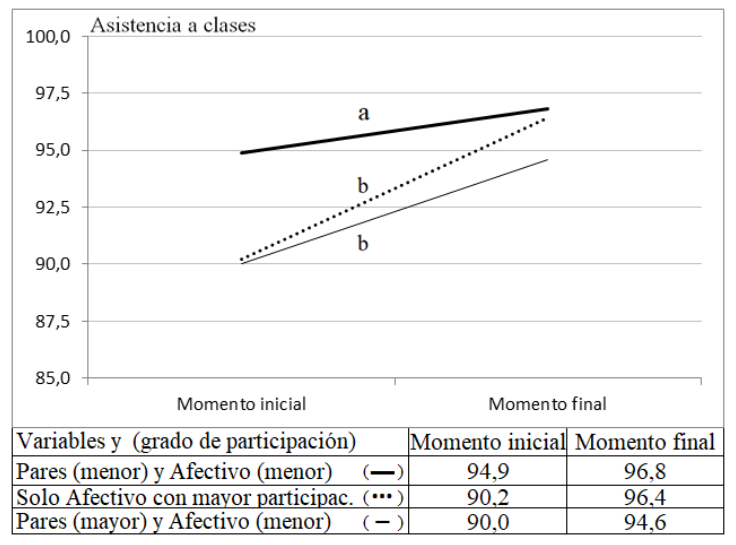

Figura 4. Gráfico de trayectorias de asistencia a clases del grupo que aumenta o mantiene su asistencia en el tiempo

A continuación, el test de contrastes ortogonales determinó que las trayectorias de los segmentos conforman dos subpoblaciones ( $\mathrm{a} \mathrm{y} \mathrm{b}$ ) estadísticamente diferentes entre sí ( $p<.05)$. Este grupo en general presentó un menor nivel de énfasis tanto en el compromiso escolar afectivo, así como en la participación los pares, observándose una leve reducción en la asistencia a clases cuando se presentó un mayor énfasis en la participación de los pares, esto último determinado a través de un efecto mediado por el componente del compromiso escolar.

En el análisis de las trayectorias del porcentaje de asistencia según los segmentos CART en el grupo de estudiantes que disminuyeron su asistencia a clases, el test multivariante de Wald evidencia que existían diferencias estadísticamente significativas entre las tendencias de las trayectorias a través del tiempo ( $p<.001)$ (Figura 5). 


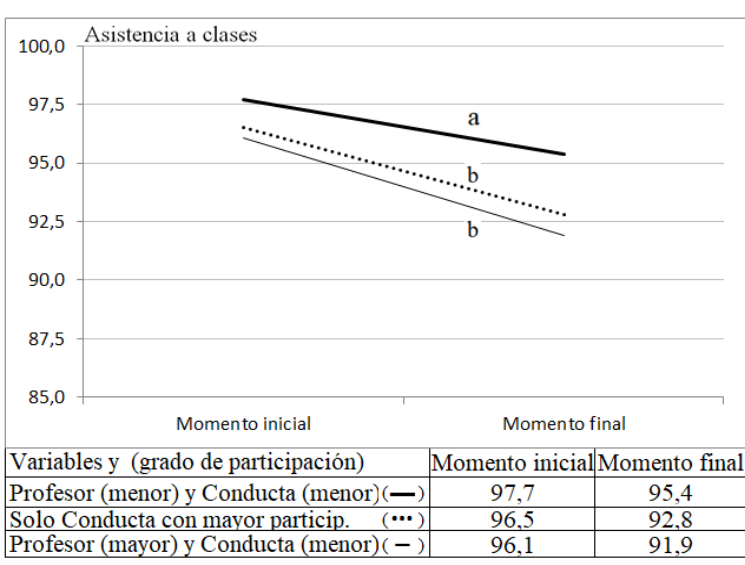

Figura 5. Gráfico de trayectorias de asistencia a clases del grupo que disminuye su asistencia en el tiempo

La aplicación del test de contrastes ortogonales evidenció que las trayectorias de los segmentos de las variables de CE y Contextuales conformaron dos subpoblaciones (a $\quad$ y b) estadísticamente diferentes entre sí $(p \leq .05)$, Este grupo en general presenta un menor nivel del compromiso escolar conductual, observándose una leve mejoría de la asistencia cuando hay una menor participación del profesorado. La más baja asistencia se presentó asociada con un aumento de la participación del profesorado. La significancia de esta última subdimensión fue estimada a través de un efecto mediado por el componente del compromiso escolar. De este modo, se puede concluir que la tercera hipótesis se corrobora, ya que fue posible evidenciar diferencias estadísticamente significativas en el análisis longitudinal de las trayectorias relacionadas con los factores del compromiso escolar y contextual sobre la diferencia de PN y AC en el tiempo.

\section{Discusión}

Generalmente en los análisis de modelos longitudinales causales, es esperable observar inestabilidad en algunos de sus componentes debido a la gran cantidad de relaciones unidireccionales y correlaciones seriales entre las varianzas residuales de las variables indicadores a través del tiempo (Newsom, 2015; Rahmadi et al., 2018). Bajo estas condiciones, los análisis de modelos SEM longitudinales presentan fuertes exigencias en la estimación de parámetros. A diferencia de lo anterior, el SEM evaluado en este estudio presenta alto ajuste psicométrico sobre los antecedentes contextuales y las subdimensiones del compromiso escolar y en la confluencia total del modelo sobre las variables de expresión del rendimiento escolar a través del tiempo.

Los resultados del modelo SEM longitudinal, muestran altos niveles de bondad de ajuste y una alta precisión de los residuales provenientes de la diferencia entre la estructura de la matriz teórica y la matriz empírica proveniente de los datos recogidos en la muestra.

El análisis transversal del rendimiento escolar de los y las estudiantes que presenta el mayor aumento en el promedio de notas en el tiempo, corresponde a aquellos y aquellas con más independencia de la familia y del profesorado. Las diferencias de nivel intermedio y menor de notas corresponden a estudiantes con mayor participación de la familia y menor participación del profesorado, esto evidencia la importancia del factor contextual familiar. Los y las estudiantes que disminuyen su promedio de notas entre los dos momentos, presentan el menor compromiso afectivo, ésta subdimensión del compromiso escolar debe ser priorizada y enfatizada para recuperar los estudiantes con menor rendimiento escolar. En aquellos y aquellas estudiantes con diferencias en el promedio de notas inferior o cercana a suficiente, el factor familiar se relacionó en forma directa con la mejoría del rendimiento escolar. Este resultado es consistente con la literatura disponible, que resalta la relevancia del contexto en el rendimiento escolar (Lara \& Saracostti, 2019; Saracostti, Lara, Martella, Miranda, Miranda-Zapata, \& Reininger, 2019).

$\mathrm{El}$ análisis longitudinal de las trayectorias del promedio de las notas a través del tiempo evidencia que las tendencias crecientes se presentan asociadas con el factor contextual familiar seguida del profesorado. Dentro de las trayectorias decrecientes, las más cercanas a la mantención de la nota, nuevamente corresponde al factor familiar y el compromiso escolar afectivo. La trayectoria más negativa se asocia con el menor compromiso escolar afectivo. Estas evidencias refuerzan la necesidad de poner énfasis en el compromiso escolar afectivo, además se debe considerar que las significancias de los factores contextuales se estiman a través de un efecto mediado por el componente del compromiso escolar. 
El análisis longitudinal de las trayectorias del porcentaje de asistencia a clases mostró que el mayor aumento se relaciona con el compromiso escolar afectivo junto con el factor contextual relacionado con los pares. Las tendencias que evidencian una disminución de la asistencia a clases se presentan asociadas a la díada conformada por un menor compromiso escolar conductual y una mayor participación de la subdimensión contextual del profesorado.

Las evidencias obtenidas en este estudio permiten identificar y priorizar las subdimensiones del compromiso escolar afectivo y los factores contextuales de la familia y el profesorado como determinantes del rendimiento escolar en su globalidad. A su vez, las asociaciones entre el compromiso escolar conductual y el factor contextual relacionado con los pares se presentan relacionados asociadas con la asistencia a clases en el tiempo. Se debe hacer notar que las significancias de los factores contextuales se encuentran mediadas por el compromiso escolar en su efecto sobre el promedio de notas y la asistencia a clases.

Finalmente, cabe mencionar algunas limitantes del presente estudio para ser solventadas en futuras investigaciones., En primer lugar, se recomienda que futuros estudios incluyan períodos de tiempo más extensos, para ampliar la evaluación de la estabilidad de las trayectorias. En segundo, sería recomendable ampliar la cantidad de regiones estudiadas lo que permitiría disponer de una muestra de mayor tamaño en relación con la cantidad de variables indicadoras del modelo SEM longitudinal. En tercer lugar, reemplazar el diseño de muestreo por conveniencia por un procedimiento probabilístico aleatorizado permitiría reducir sesgos de selección. Finalmente, en cuanto a la personalización de los casos, se sugiere considerar la incorporación de una medición de destrezas socio adaptativas que podrían presentar relación con el compromiso escolar y factores contextuales.

\section{Financiamiento}

Este artículo ha recibido el apoyo de los proyectos ANID/FONDECYT 1210172, FONDEF ID14I20078 y FONDEF IT 19I0012", financiados por la Comisión Nacional de Investigación Científica y Tecnológica CONICYT, Chile.

\section{Referencias}

Asociación Chilena De Municipalidades (ACHM), San Joaquín Educa, Municipalidad De Estación Central, Peñalolén, Caleta Sur, Centro De Estudios De La Niñez, Fundación Súmate Del Hogar De Cristo, UNICEF. (2013). La exclusión educativa un desafio urgente para Chile: Propuestas para ampliar las capacidades de inclusión del sistema educativo chileno. Recuperado de https://sanjoaquineduca.cl/wp-

content/uploads/2013/10/La_exclusion_educat iva_desafio_urgente_para_Chile.pdf

Asociación Chilena Pro Naciones Unidas, ACHNU. (2006). La deserción escolar en Chile ¿Prioridad en la agenda educativa?. Recuperado de http://www.redligare.org/IMG/pdf/desercion_ escolar_chile_flamey.pdf

Centro de Estudios del MINEDUC. (2014). Serie evidencias: medición de la deserción escolar en Chile, 2(15), 1-11. Recuperado de http://centroestudios.mineduc.cl/tp_enlaces/po rtales/tp5996f8b7cm96/uploadImg/File/A15N 2_Desercion.pdf

Comisión Económica para América Latina y el Caribe, CEPAL. (2015). Hacia la inclusión social juvenil: Herramientas para el análisis y el diseño de políticas. Santiago. Recuperado de

https://www.cepal.org/es/publicaciones/39001 -la-inclusion-social-juvenil-herramientasanalisis-diseno-politicas

Espinoza-Díaz, Ó., Castillo-Guajardo, D., González, L. E., Loyola-Campos, J., \& CruzGrau, E. (2014). Deserción escolar en Chile: un estudio de caso en relación con factores intraescolares. Educación y Educadores, 17(1), 32-50. doi:10.5294/edu.2014.17.1.2

Fernández-Zabala, A., Goñi, I., \& Zulaika, L.M. (2015). Family and school context in school engagement. European Journal of Education and Psychology, 9(2), 47-55.

doi:10.1016/j.ejeps.2015.09.001

Gorostiaga, A., Balluerka, N., Ulaciay I., \& Aliri, J. (2017). Evaluación de la iniciativa personal en el ámbito educativo y su relación con la actitud emprendedora y el rendimiento académico. Revista Iberoamericana de 
Diagnóstico y Evaluación - e Avaliação

Psicológica, 3(48), 105-116.

doi:10.21865/RIDEP48.3.09

JUNAEB. (2015). Caracterización de la vulnerabilidad de niños y niñas en establecimientos de educación parvularia 2015. Recuperado de https://www.junaeb.cl/wpcontent/uploads/2018/08/Caracterizaci\%C3\% B3n-de-la-Vulnerabilidad-en-Parvularia2015.pdf

Kline, R. B. (2010). Principles and practice of structural equation modeling ( $3^{\text {rd }}$ ed.). New York: The Guilford Press.

Lara, L., \& Saracostti, M. (2019). Effect of parental involvement on children's academic achievement in Chile. Frontiers in Psychology, 10, 1-5. doi: 10.3389/fpsyg.2019.01464

Lara, L., Saracostti, M., Navarro, J. J., de-Toro, X., Miranda-Zapata, E., Trigger, J. M., \& Fuster, J. (2018). Compromiso escolar: Desarrollo y validación de un instrumento. Revista Mexicana de Psicología, 35(1), 52-62.

Marsh, H. W., Hau, K. T., \& Grayson, D. (2005). Goodness of fit evaluation in structural equation modeling. En A. Maydeu-Olivares, y J. McArdle (Eds.), Contemporary psychometrics. A Festschrift for Roderick $P$. McDonald. (pp. 275-340). Lawrence Erlbaum Associates Publishers.

Melo, G., Failache, E., \& Machado, A. (2015). Adolescents that do not attend junior high: characterization of their academic trajectory, living conditions and decision to dropout. Páginas de Educación, 8(2), 66-88. Recuperado de http://www.scielo.edu.uy/scielo.php?script=sc i_arttext\&pid=S1688-

$74682015000200003 \& \operatorname{lng}=\mathrm{es} \& \operatorname{tlng}=\mathrm{es}$

Miranda-Zapata, E., Lara, L., Navarro, J.-J., Saracostti, M., \& de-Toro, X. (2018). Modelización del efecto del Compromiso Escolar sobre la Asistencia a clases y el Rendimiento escolar en una muestra de estudiantes chilenos. Revista de Psicodidáctica, 23(2), 102-109. doi:10.1016/j.psicod.2018.02.003

Newsom, J. T. (2015). Multivariate applications series.Longitudinal structural equation modeling: A comprehensive introduction. New York: Routledge/Taylor \& Francis Group.

Rahmadi, R., Groot, P., van Rijn, M. H., van den Brand, J. A., Heins, M., Knoop, H., Heskes, T., \& OPTIMISTIC Consortium. (2018). Causality on longitudinal data: Stable specification search in constrained structural equation modeling. Statistical Methods in Medical Research, 27(12), 3814-3834. doi:10.1177/0962280217713347

Saracostti, M., Lara, L., Martella, D., Miranda, H., Miranda-Zapata, E. D., \& Reininger, T. (2019). Influence of family involvement and children's socioemotional development on the learning outcomes of Chilean students. Frontiers in Psychology, 10, 1-7. doi:10.3389/fpsyg.2019.00335.

Vera-Bachmann, D., Gálvez-Nieto, J. L., TrizanoHermosilla, Í., \& Álvarez-Espinoza, A. (2020). Estudio psicométrico del Inventario para la Planificación de Servicios y la Programación Individual (ICAP), en población de estudiantes chilenos. Revista Iberoamericana de Diagnóstico y Evaluación Psicológica, 1(54), 119-129. doi:10.21865/RIDEP54.1.10

Wylie, C., \& Hodgen, E. (2012). Trajectories and patterns of student engagement: Evidence from a longitudinal study. En S. L. Christenson, A. L. Reschly, y C. Wylie (Eds.), Handbook of research on student engagement (pp. 585-599). New York: Springer. doi:10.1007/978-1-4614-2018-7

\section{Anexo 1.}


Estimaciones de los modelos de medida

\begin{tabular}{|c|c|c|c|c|c|c|c|c|}
\hline & \multicolumn{4}{|c|}{ Momento incial } & \multicolumn{4}{|c|}{ Momento final } \\
\hline $\begin{array}{c}\text { Ítems C. E. } \\
\text { Afectivo }\end{array}$ & Est*. & $\begin{array}{l}\text { Error } \\
\text { est. }\end{array}$ & $\begin{array}{c}\text { Est./Error } \\
\text { est. }\end{array}$ & P-valor & Est. & $\begin{array}{l}\text { Error } \\
\text { est. }\end{array}$ & $\begin{array}{c}\text { Est./Error } \\
\text { est. }\end{array}$ & P-valor \\
\hline V1 & 0.67 & 0.04 & 16.05 & 0.00 & 0.69 & 0.04 & 18.26 & 0.00 \\
\hline $\mathrm{V} 2$ & 0.67 & 0.04 & 15.18 & 0.00 & 0.59 & 0.04 & 13.41 & 0.00 \\
\hline V3 & 0.68 & 0.04 & 15.88 & 0.00 & 0.71 & 0.03 & 21.55 & 0.00 \\
\hline V4 & 0.77 & 0.04 & 20.18 & 0.00 & 0.63 & 0.04 & 15.78 & 0.00 \\
\hline V5 & 0.67 & 0.04 & 17.26 & 0.00 & 0.80 & 0.03 & 28.50 & 0.00 \\
\hline V6 & 0.75 & 0.04 & 19.74 & 0.00 & 0.81 & 0.03 & 28.96 & 0.00 \\
\hline V7 & 0.77 & 0.03 & 22.76 & 0.00 & 0.74 & 0.04 & 20.03 & 0.00 \\
\hline V8 & 0.55 & 0.06 & 9.55 & 0.00 & 0.73 & 0.03 & 21.32 & 0.00 \\
\hline V9 & 0.76 & 0.04 & 21.06 & 0.00 & 0.76 & 0.03 & 23.81 & 0.00 \\
\hline V10 & 0.80 & 0.03 & 24.91 & 0.00 & 0.84 & 0.02 & 38.36 & 0.00 \\
\hline $\begin{array}{c}\text { Ítems C. E. } \\
\text { Cognitivo }\end{array}$ & Est*. & $\begin{array}{l}\text { Error } \\
\text { est. }\end{array}$ & $\begin{array}{c}\text { Est./Error } \\
\text { est. }\end{array}$ & P-valor & Est. & $\begin{array}{l}\text { Error } \\
\text { est. }\end{array}$ & $\begin{array}{c}\text { Est./Error } \\
\text { est. }\end{array}$ & P-valor \\
\hline V11 & 0.62 & 0.05 & 12.88 & 0.00 & 0.57 & 0.04 & 12.98 & 0.00 \\
\hline V12 & 0.68 & 0.05 & 14.45 & 0.00 & 0.58 & 0.05 & 12.06 & 0.00 \\
\hline V13 & 0.77 & 0.04 & 18.66 & 0.00 & 0.69 & 0.04 & 19.14 & 0.00 \\
\hline V14 & 0.59 & 0.05 & 11.65 & 0.00 & 0.68 & 0.05 & 15.00 & 0.00 \\
\hline V15 & 0.53 & 0.06 & 9.35 & 0.00 & 0.61 & 0.05 & 12.75 & 0.00 \\
\hline V16 & 0.72 & 0.04 & 18.87 & 0.00 & 0.76 & 0.03 & 23.72 & 0.00 \\
\hline V17 & 0.53 & 0.05 & 10.33 & 0.00 & 0.51 & 0.05 & 9.90 & 0.00 \\
\hline V18 & 0.74 & 0.05 & 16.51 & 0.00 & 0.81 & 0.04 & 23.20 & 0.00 \\
\hline V19 & 0.68 & 0.04 & 16.93 & 0.00 & 0.68 & 0.04 & 17.41 & 0.00 \\
\hline V20 & 0.72 & 0.04 & 19.49 & 0.00 & 0.62 & 0.04 & 15.20 & 0.00 \\
\hline V21 & 0.65 & 0.04 & 15.21 & 0.00 & 0.74 & 0.04 & 20.47 & 0.00 \\
\hline V22 & 0.66 & 0.05 & 14.02 & 0.00 & 0.75 & 0.04 & 20.14 & 0.00 \\
\hline $\begin{array}{l}\text { Ítems C. E. } \\
\text { Conductual }\end{array}$ & Est*. & $\begin{array}{l}\text { Error } \\
\text { est. }\end{array}$ & $\begin{array}{c}\text { Est./Error } \\
\text { est. }\end{array}$ & P-valor & Est. & $\begin{array}{c}\text { Error } \\
\text { est. }\end{array}$ & $\begin{array}{c}\text { Est./Error } \\
\text { est. }\end{array}$ & P-valor \\
\hline V23 & 0.58 & 0.16 & 3.61 & 0.00 & 0.51 & 0.11 & 4.59 & 0.00 \\
\hline V24 & 0.69 & 0.15 & 4.49 & 0.00 & 0.55 & 0.11 & 4.80 & 0.00 \\
\hline V25 & 0.55 & 0.11 & 5.08 & 0.00 & 0.61 & 0.10 & 6.30 & 0.00 \\
\hline V26 & 0.81 & 0.10 & 8.47 & 0.00 & 0.77 & 0.07 & 10.68 & 0.00 \\
\hline V28 & 0.55 & 0.11 & 5.20 & 0.00 & 0.64 & 0.09 & 7.04 & 0.00 \\
\hline V29 & 0.52 & 0.10 & 5.06 & 0.00 & 0.75 & 0.07 & 10.35 & 0.00 \\
\hline
\end{tabular}

*Est.=Estimación

\section{Anexo 2.}

\section{Estimaciones de las saturaciones de los ítems en el Factor Contextual Familia}




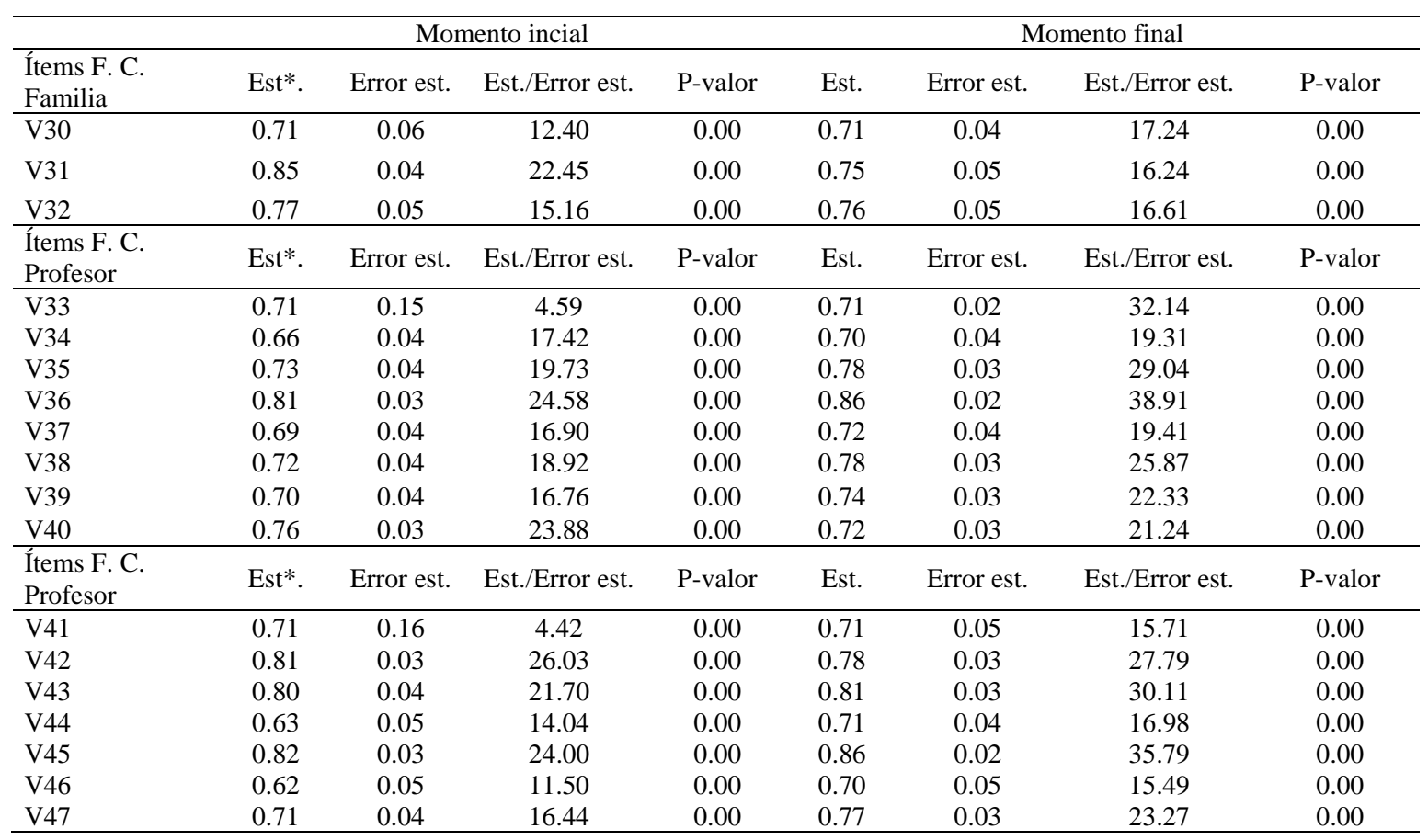

\section{Anexo 3.}

Path del Modelo SEM longitudinal en el momento inicial

\begin{tabular}{|c|c|c|c|c|c|c|c|c|}
\hline \multirow[b]{2}{*}{ Path: Afectivo } & \multicolumn{4}{|c|}{ Momento inicial } & \multicolumn{4}{|c|}{ Momento final } \\
\hline & Est. & Err.est. & Est/Err.est. & P-valor & Est. & Err.est. & Est/Err.est. & P-valor \\
\hline Familia & 0.14 & 0.01 & 14.10 & 0.00 & 0.21 & 0.06 & 3.31 & 0.00 \\
\hline Profesor & 0.79 & 0.06 & 12.68 & 0.00 & 0.77 & 0.06 & 13.19 & 0.00 \\
\hline Pares & 0.06 & 0.01 & 6.00 & 0.00 & 0.10 & 0.01 & 10.00 & 0.00 \\
\hline Path: Cognitivo & Est. & Err.est. & Est/Err.est. & P-valor & Est. & Err.est. & Est/Err.est. & P-valor \\
\hline Familia & 0.14 & 0.03 & 4.70 & 0.00 & 0.56 & 0.09 & 6.06 & 0.00 \\
\hline Profesor & 0.79 & 0.06 & 12.68 & 0.00 & 0.83 & 0.10 & 8.11 & 0.00 \\
\hline Pares & 0.06 & 0.01 & 6.00 & 0.00 & -0.56 & 0.12 & -4.79 & 0.00 \\
\hline Path: Conducta & Est. & Err.est. & Est/Err.est. & $\mathrm{P}$-valor & Est. & Err.est. & Est/Err.est. & P-valor \\
\hline Familia & 0.14 & 0.03 & 4.70 & 0.00 & 0.50 & 0.13 & 3.75 & 0.00 \\
\hline Profesor & 0.79 & 0.06 & 12.68 & 0.00 & 0.69 & 0.15 & 4.67 & 0.00 \\
\hline Pares & 0.06 & 0.01 & 6.00 & 0.00 & -0.73 & 0.17 & -4.44 & 0.00 \\
\hline $\begin{array}{l}\text { Path: Promedio de } \\
\text { notas }\end{array}$ & Est. & Err.est. & Est/Err.est. & $\mathrm{P}$-valor & Est. & Err.est. & Est/Err.est. & P-valor \\
\hline Afectivo & 0.09 & 0.02 & 4.25 & 0.00 & -0.23 & 0.02 & -11.50 & 0.00 \\
\hline Cognitivo & -0.14 & 0.03 & -4.67 & 0.00 & 0.52 & 0.09 & 5.95 & 0.00 \\
\hline Conducta & 0.22 & 0.03 & 7.47 & 0.00 & 0.35 & 0.07 & 5.00 & 0.00 \\
\hline $\begin{array}{l}\text { Path: Asistencia a } \\
\text { clases }\end{array}$ & Est. & Err.est. & Est/Err.est. & P-valor & Est. & Err.est. & Est/Err.est. & P-valor \\
\hline Afectivo & -0.13 & 0.02 & -6.30 & 0.00 & -0.08 & 0.02 & -4.15 & 0.00 \\
\hline Cognitivo & 0.36 & 0.09 & 4.09 & 0.00 & 0.14 & 0.02 & 6.80 & 0.00 \\
\hline Conducta & 0.44 & 0.06 & 6.94 & 0.00 & 0.20 & 0.05 & 4.06 & 0.00 \\
\hline
\end{tabular}

